\title{
Navigating Your Acute Heart Failure Patient in Emergency and Pre- Discharge Phase
}

\author{
Budi Yuli Setianto \\ Department of Cardiology and Vascular Medicine, Faculty of Medicine, Public Health and Nursing, Universitas Gadjah Mada - \\ Dr. Sardjito General Hospital, Yogyakarta, Indonesia
}

Corresponding author:

Budi Yuli Setianto, MD, - email: budyuls@ugm.ac.id

Department of Cardiology and Vascular Medicine, Faculty of Medicine, Public Health and Nursing,Universitas Gadjah Mada Dr. Sardjito Hospital

Jalan Farmako, Sekip Utara, Yogyakarta, Indonesia 55281

\section{ABSTRACT}

Heart failure (HF) leads to frequent hospitalizations. The presence of rehospitalization risk among patientshospitalized for heart failure is important, especially hemodynamic instability and neurohormonal over activation. ARNI is needed to restore the balance of neurohormonal system in HF. PARADIGM-HF study provide insight on long term benefit of ARNI (i.e. sacubitril/valsartan) in ambulatory setting. How is the evidence of ARNI use for in-hospitalization phase of HF? PIONEER and TRANSITION showed that initiation of sacubitril/valsartan shortly after an ADHF event is feasible and well tolerated. In-hospital initiation of sacubitril/valsartan is associated with early and sustained improvements in biomarkers of cardiac wall stress and myocardial injury, indicating pathophysiological benefits in a wide range of HFrEF patients.

Keywords: ambulatory heart failure; acute heart failure; ARNI

\section{Introduction}

Heart failure (HF) frequently leads to hospitalization. HF is one of the leading causes of hospitalization among patients aged > 65 years old in developed countries. ${ }^{1}$ Approximately $44 \%$ of all $\mathrm{HF}$ patients had hospital readmissions due to any causes within 1 year after discharge. ${ }^{2}$ Length of hospital stay for HF is ranging from 5 to 10 days. $^{3}$ In the United States of America, hospital readmission rate within 30 days after discharge is $25 \%,{ }^{4}$ meanwhile in European countries, hospital readmission rate is $24 \%$ within 12 weeks after discharge. ${ }^{5}$

\section{Discussion}

Re-hospitalization risk among patients who were hospitalized for heart failure happened especially during patient's transition of care period, which is a periodof shifting from close supervision by cardiologist team at the hospital to outpatient monitoring (which is less frequent compared to in-hospital monitoring) at home after discharge. Rehospitalization risk will remain high if there are hemodynamic imbalance and neurohormonal over activation. ${ }^{6,7}$ Nearly $25 \%$ of HF patients will be readmitted within the first 30 days after discharge. The mortality rate during the 30-day period can be as high as $10 \%{ }^{6}$ The number of death associated with time after discharge is twice higher in the first 30 days compared to 6 months after discharge. (Figure 1) 


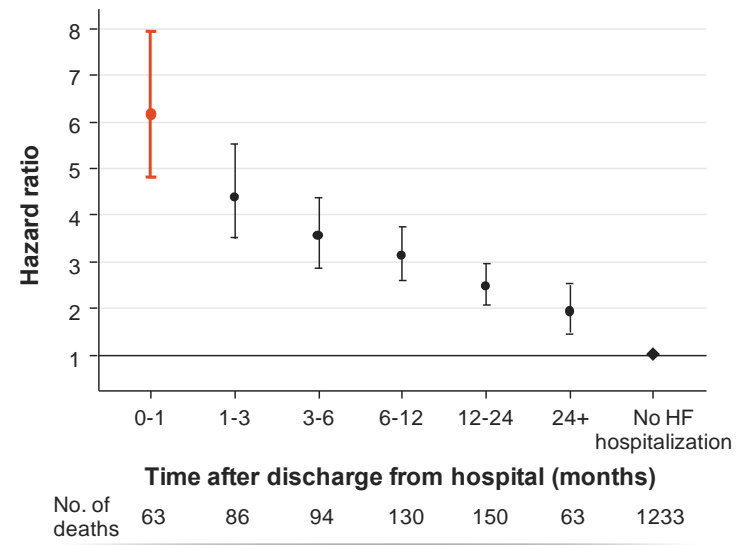

Figure 1. Mortality risk is twice higher within the first 30 days compared to 6 months after hospital discharge. ${ }^{6}$

ARNI on neurohormonal system balance in $\mathrm{HF}$

Prospective comparison of ARNI with ACEl to Determine of impact on Global Mortality and morbidity in Heart Failure (PARADIGM-HF) study provides insight to long-term benefits of $A R N I$ in outpatient setting. PARADIGM-HF study result showed that sacubitril/valsartan is superior to enalapril. In addition to it, PARADIGM-HF study showed reduction of mortality risk due to cardiovascular events or first HF hospitalization for $20 \%{ }^{8}$

Whether the initiation of sacubitril/valsartan therapy is effective and safe among hospitalized patients due to acute decompensated heart failure still remains unknown. The following study will acknowledge this question further.The Comparison of Sacubitril/Valsartan Versus Enalapril on Effect on NT-ProBNP in Patients Stabilized from an Acute Heart Failure Episode (PIONEER-HF) study examined heart failure patients with reduced ejection fraction (HFrEF) who were hospitalized due to acute decompensated heart failure in 129 locations in the United States of America. After hemodynamic stabilization, eligible patients were randomized in a 1:1 fashion to sacubitril/valsartan group (targeted dose of $97 \mathrm{mg}$ sacubitril with $103 \mathrm{mg}$ valsartan twice daily) and enalapril group (targeted dose of $10 \mathrm{mg}$ enalapril twice daily). The primary efficacy outcome is a time-averaged proportional change of $n$ terminal pro-B-type natriuretic peptide (NT-proBNP) from the beginning of the study until week 4 and week 8 . The main safety outcomes are worsening renal function, hyperkalemia, symptomatic hypotension, and angioedema. Of 881 eligible patients who underwent randomization, 440 patients received sacubitril/valsartan while 441 patients received enalapril. Time-averaged reduction of NT-proBNP level was significantly greater insacubitril/valsartan group than enalapril group. Geometric mean ratio of values obtained at week 4 and week 8 from baseline was 0,53 in sacubitril/valsartan group compared to 0.75 in enalapril group (percent change, $46.7 \%$ vs $-25.3 \%$; change ratio of sacubitril/valsartan group compared to enalapril group $0.71 ; 95 \%$ confidence interval $(\mathrm{Cl}) 0.63$ to $0.81 ; \mathrm{P}<0.001)$. At the beginning of week 1 after drug initiation, a greater reduction of NT-proBNP level in sacubitril/valsartan group than in enalapril group was noted (change ratio 0.76; 95\% $\mathrm{Cl} 0.69$ to 0.85 ). Worsening renal function, hyperkalemia, symptomatic hypotension, and angioedema did not differ significantly between both groups. ${ }^{9}$ (Figure 2 and 3). 


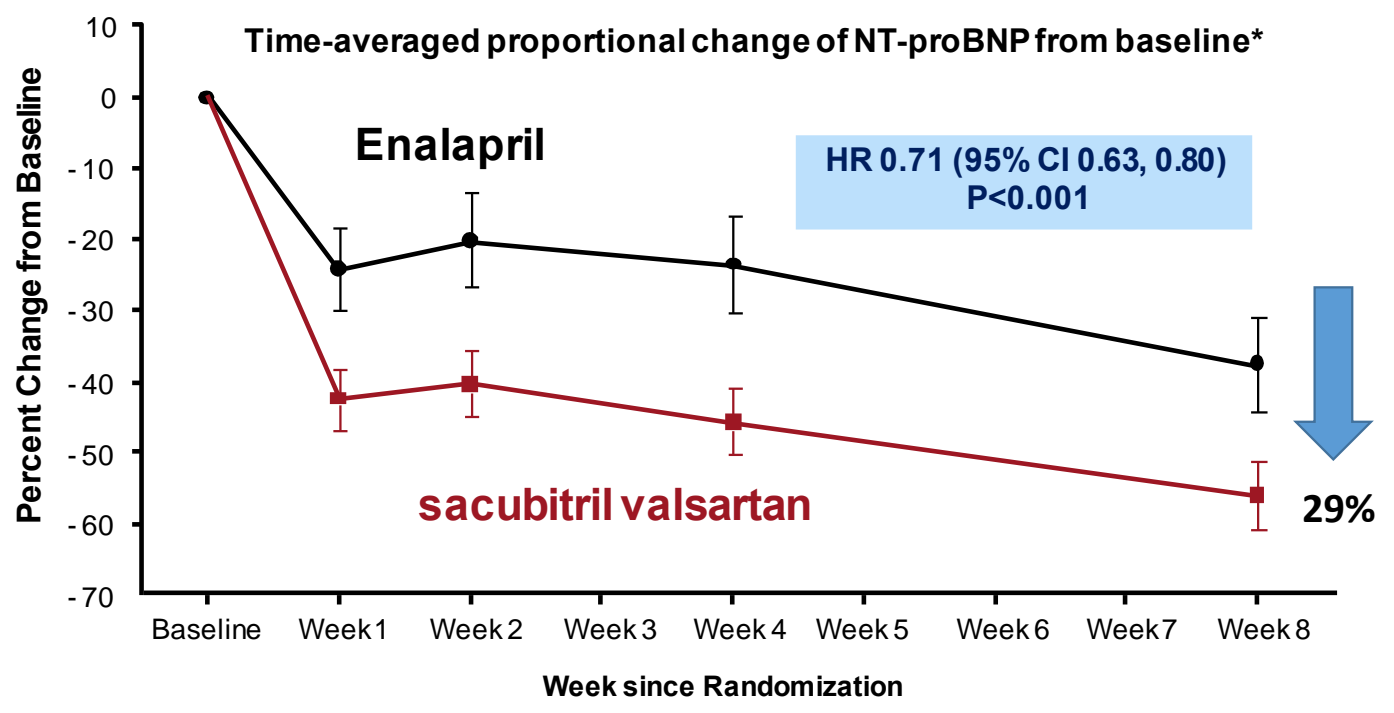

Figure 2. In-hospital initiation of sacubitril/valsartan decreases level of NT-pro BNP to a greater degree than enalapril. ${ }^{6}$

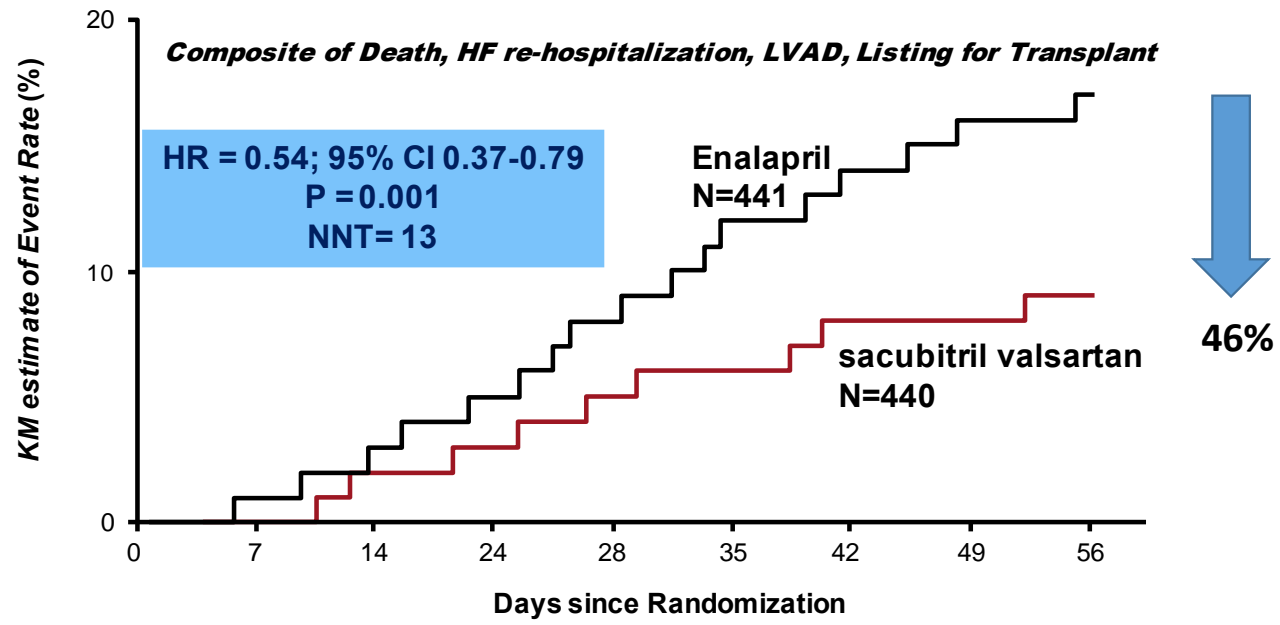

- Exploratory Serious Clinical Composite endpoint was driven by the reduction of risk of death and HF re-hospitalizations

Figure 3. In-hospital initiation of Sacubitril/Valsartan reduces serious clinical outcomes compared to enalapril. ${ }^{6}$

In-hospital initiation of sacubitril/valsartan with HFrEF after hemodynamic stabilization was reported in the main outcome of TRANSITION study. In this study, $50 \%$ of patients achieved targeted dose of $200 \mathrm{mg}$ twice a day in 10 weeks, and more than $85 \%$ of patients could receive the targeted dose without interruption in both groups (pre-discharge and post-discharge) (Figure 4). ${ }^{10}$ Predictors of success of sacubitril/valsartan up-titration to $200 \mathrm{mg}$ were younger, healthier, de novo, and hypertensive patients. 


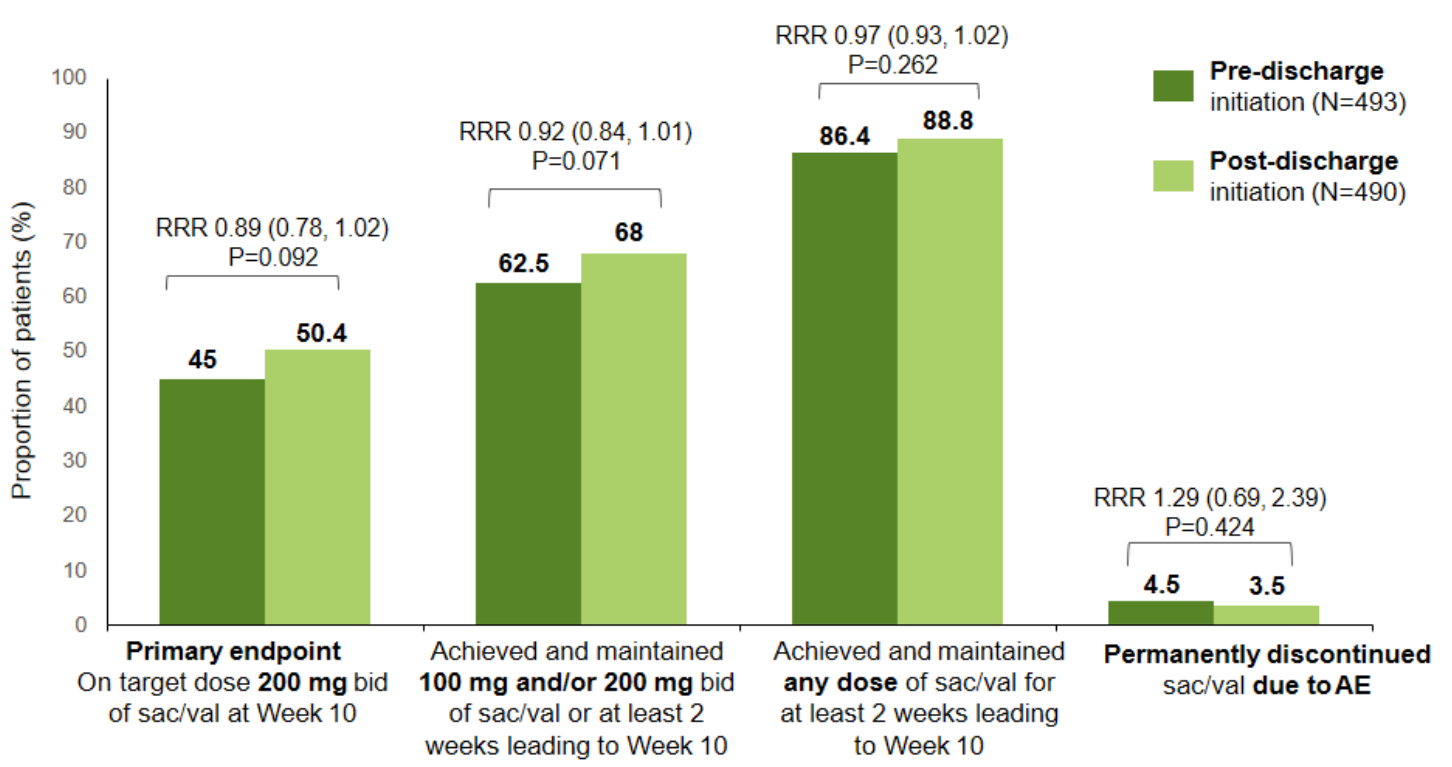

Figure 4. Primary and secondary endpoints from TRANSITION study. ${ }^{10}$

\section{Conclusion}

Among heart failure patients with reduced ejection fraction who were hospitalized for acute decompensated heart failure, in-hospital initiation of sacubitril/valsartan was associated with greater reduction of NT-proBNP level than enalapril. Worsening renal function, hyperkalemia, symptomatic hypotension, and angioedema did not differ significantly between sacubitril/valsartan group and enalapril group.

\section{References}

1. Bui A.L., Horwich T.B., Fonarow G.C. 2011. Epidemiology and risk profile of heart failure. Nat Rev Cardiol, 8:3041.

2. Maggioni A.P., Dahlström U., Filippatos G., Chioncel O., Crespo Leiro M., Drozdz J., et al. 2013. Heart Failure Association of the European Society of Cardiology (HFA).EURObservational Research Programme: regional differences and 1-year follow-up results of the Heart Failure Pilot Survey (ESC-HF Pilot). Eur J Heart Fail, 15:808-817.
3. Ponikowski P., Anker S.D., AlHabib K.F., Cowie M.R., Force T.L., Hu S., et al. 2014. Heart failure: preventing disease and death worldwide. ESC Heart Fail, 1:4-25.

4. Kociol R.D., LiLiang, Hernandez A.F., Heidenreich P.A., Yancy C.W., Fonarow G.C., et al. 2013. Are we targeting the right metric for heart failure? Comparison of hospital 30-day readmission rates and total episode of care inpatient days. Am Heart J, 165:987-994.

5. Cleland J.G.F., $\quad$ Swedberg K., Follath F., Komajda M., CohenSolal A., Aguilar J.C., et al. 2003. The EuroHeart Failure survey programme-a survey on the quality of care among patients with heart failure in Europe: Part 1: patient characteristics and diagnosis. Eur Heart J, 24:442-463.

6. Greene S.J., Fonarow G.C., Vaduganathan M., Khan S.S., Butler J., Gheorghiade M. 2015. The vulnerable phase after hospitalization for heart failure. Nat Rev Cardiol, 12:220-229.

7. Langenickel T.H, Dole W.P. 2012. Angiotensin receptor-neprilysin inhibition with LCZ696: a novel approach for the treatment of heart 
failure. Drug Discov Today: Therapeutic Strategies, 9:e131-e139.

8. McMurray J.J.V., Packer P., Desai A.S., Gong J., Lefkowitz M.P., Rizkala A.R., et al. 2014. for the PARADIGMHF Investigators and Committees. Angiotensin-Neprilysin Inhibition versus Enalapril in Heart Failure. N Engl J Med, 371:993-1004.

9. Velazquez E.J., Morrow D.A., DeVore A.D., Duffy C.I., Ambrosy A.P., McCague K., et al. 2019. Angiotensin-
Neprilysin inhibition in acute decompensated heart failure. $\mathrm{N}$ Engl $\mathrm{J}$ Med, 380:539-548.

10. Wachter R. 29018. Initiation of sacubitril/valsartan in hospitalized patients with heart failure with reduced ejection fraction after hemodynamic stabilization: primary results of the TRANSITION study. TRANSITION primary data poster presentation (P886) at ESC Congress 2018, Munich Germany. 